\title{
LETTERS
}

\section{An unusual cause of blindness}

\section{To the Editors:}

Allergic bronchopulmonary aspergillosis (ABPA) is caused by colonisation of the lungs with Aspergillus fumigatus resulting in a hypersensitivity reaction and bronchiectasis. Allergic aspergillus sinusitis (AAS) has similar histopathological features and yet simultaneous occurrence is rarely reported. We report a case of an aspergilloma in the paranasal sinuses causing unilateral blindness with associated AAS. The patient went on to develop ABPA resulting in a dramatic decline in lung function. Therapy consisted of oral steroids, an anti-fungal agent, bronchodilators and bronchial hygiene.

A 48-yr-old female presented to her general practitioner in 2006 with sudden loss of sight in the left eye. Following an urgent computed tomography (CT) head scan she was referred to the Ear Nose and Throat surgeons (Royal Infirmary Edinburgh, Edinburgh, UK) with the radiological diagnosis of a cystic lesion in the region of the sphenoethmoid sinus (fig. 1). She underwent an endoscopic sphenoid sinus debridement and washout. Cellular pathology of the sinus biopsy showed an oedematous and hyalinised lamina propria, containing an infiltrate of plasma cells, eosinophils and lymphoid aggregates. Branching septate fungal hyphae consistent with Aspergillus sp. were identified. The patient was diagnosed with an aspergillus fungal ball and sinusitis. Full

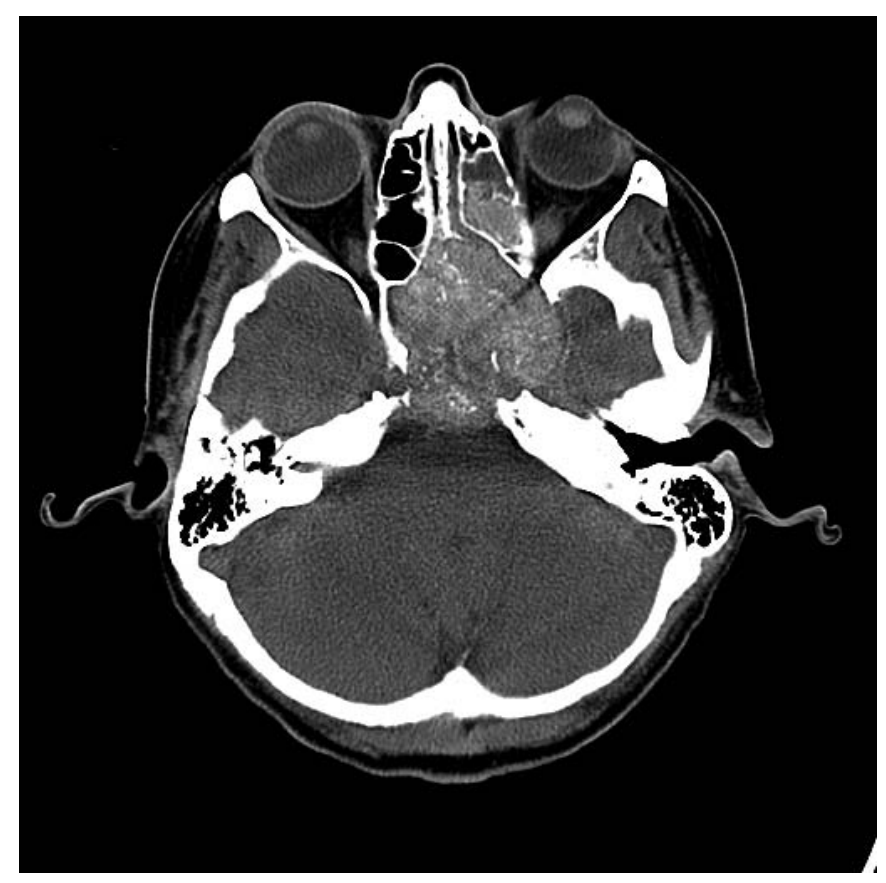

FIGURE 1. Computed tomography head scan of the axial section showing large aspergilloma invading the sphenoethmoid sinus and extending posteriorly with compression of the left optic nerve. vision was restored completely and no further treatment was instituted.

Later that year she presented with worsening exertional breathlessness and wheeze, a productive cough with mucous plugs and weight loss (body mass index $17.7 \mathrm{~kg} \cdot \mathrm{m}^{-2}$ ). She had history of severe asthma since childhood during which time she had been admitted to hospital several times, once requiring ventilation. In adulthood she reported reasonable asthma control apart from an admission for pneumonia in 1999 and had never been diagnosed with ABPA.

On this occasion her sputum grew Aspergillus fumigatus $\left(5,000 \mathrm{cfu} \cdot \mathrm{mL}^{-1}\right)$ for the first time and a CT scan of the thorax revealed central bronchiectasis, tubular and varicose, involving all lobes with ground-glass attenuation and coarse thickened linear markings (fig. 2). She was positive for aspergillus precipitins, her total immunoglobulin (Ig)E was $>5,000 \mathrm{Ku} \cdot \mathrm{L}^{-1}$, Aspergillus $\operatorname{IgE}$ was $52.80 \mathrm{Ku} \cdot \mathrm{L}^{-1}$ and her eosinophil count was $4.1310^{9} \cdot \mathrm{L}^{-1}$. Her spirometry revealed a forced expiratory volume in $1 \mathrm{~s}$ (FEV1)/vital capacity (VC) ratio of $48 \%$ predicted with minimal reversibility. She was diagnosed with ABPA and was started on a high dose of prednisolone, inhaled steroids (beclomethasone $250 \mu \mathrm{g}$ metered dose inhaler two puffs b.i.d.), salmeterol $(25 \mu \mathrm{g}$ metered dose inhaler two puffs b.i.d.) and montelukast

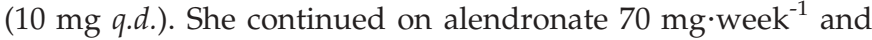
calcium/vitamin D for early menopause and osteopenia, in

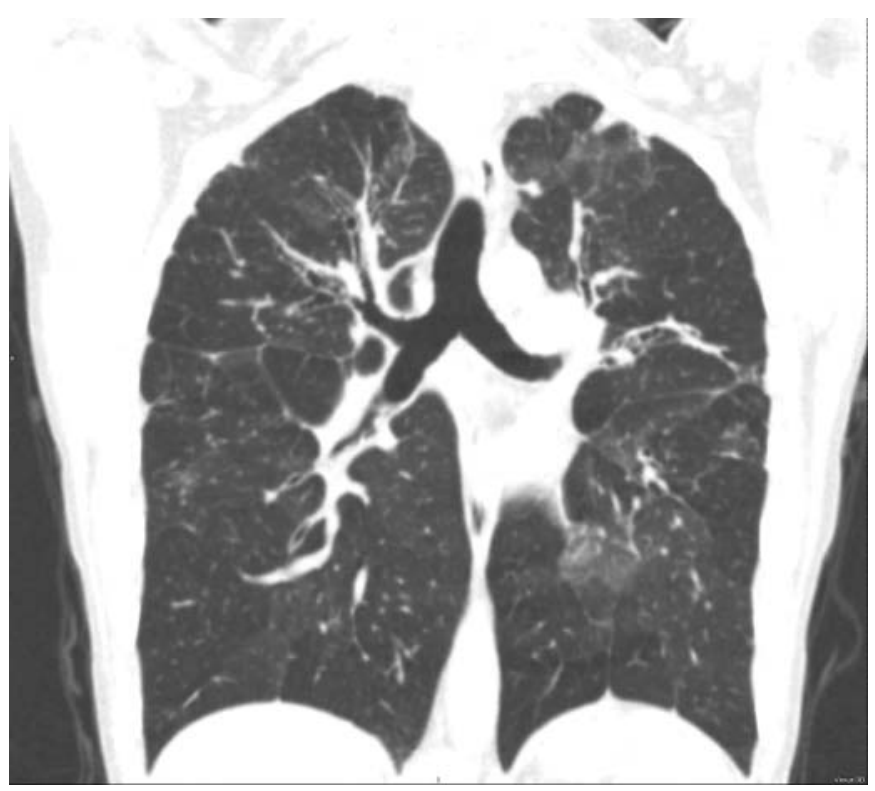

FIGURE 2. Computed tomography scan of the coronal section of the chest showing severe proximal bronchiectasis consistent with a diagnosis of allergic bronchopulmonary aspergillosis. 
addition to omeprazole $10 \mathrm{mg}$ q.d. and ferrous sulphate $200 \mathrm{mg}$ q.d. The patient was reviewed regularly in the chest clinic (Royal Infirmary Edinburgh) for recurrent chest infections, generally growing Moraxella catarrhalis susceptible to penicillin. In November 2007, she was started on itraconazole $400 \mathrm{mg}$ q.d. which was reduced to $200 \mathrm{mg}$ q.d.

After 4 months, her FEV1/VC ratio had improved to 58\% with no exacerbations of asthma and minimal sputum production. She is tolerating itraconazole with no side-effects and is gaining weight. On review in August 2009, she was continuing on itraconazole $200 \mathrm{mg}$ q.d. and inhaled steroids as mentioned previously; she has weaned herself off oral steroids completely with no decrease in respiratory function.

AAS is characterised by mucoid impaction in the paranasal sinuses with very similar histopathological features to ABPA [1]. An aspergilloma results from colonisation with Aspergillus of an existing cavity; as in our case a paranasal sinus. It is hypothesised that an aspergilloma may act as a locus for antigenic stimulation in a genetically predisposed individual resulting in the development of ABPA [2]. Although contemporaneous occurrence of ABPA, AAS and/or aspergilloma has been reported, it is very rare.

12 cases of simultaneous AAS and ABPA and just one case of AAS, ABPA and aspergilloma have been reported in the literature. A review of 12 cases of AAS associated with ABPA showed that only two patients demonstrated simultaneous AAS and ABPA [3-5]. One case of AAS and ABPA refractory to standard therapy was successfully treated with voriconizole [6]. A search for concomitant ABPA and AAS cases in India over a 10-yr period revealed just seven reported cases [7]. The same group later identified a case of a 24-yr-old male with a history of asthma and rhinitis presenting with haemoptysis who was subsequently diagnosed with concomitant AAS, aspergilloma and ABPA [8].

Our patient developed symptoms of ABPA after the AAS and aspergilloma were identified and treated.

Management of allergic sinusitis and aspergilloma/ABPA includes oral corticosteroids, antifungal therapy, bronchodilators and bronchial hygiene. Oral corticosteroids suppress the immunological and inflammatory response in ABPA, improving symptoms of bronchospasm and cough [9]. Recommended therapy is $0.5 \mathrm{mg} \cdot \mathrm{kg}^{-1} \cdot \mathrm{day}^{-1}$ for 2 weeks gradually tapered thereafter according to clinical and radiological response. Lengthy therapy may be required to prevent relapse. Nebulised corticosteroid and amphotericin B have also shown some efficacy in treating ABPA.

Itraconazole reduces inflammation and improves clinical outcome resulting in less frequent exacerbations in patients with ABPA. A daily dose of $400 \mathrm{mg}$ for 16 weeks resulted in clinical improvement for $46 \%$ of patients in a double-blind randomised controlled trial and a significant decline in $\operatorname{IgE}$ levels and sputum eosinophil count [10]. One additional treatment that is available for ABPA is omalizumab, a recombinant anti-IgE antibody which has been shown to be efficacious in patients with cystic fibrosis and ABPA, and in severe asthma unresponsive to standard medication, allowing reduction of steroid therapy [11]. Owing to her persistently elevated IgE levels, our patient has never met the criteria for the use of omalizumab and has so far responded remarkably to long-term itraconazole use with stabilisation of her disease process.

\section{R.A. Seville*, J. Murchison ${ }^{\#}$ and R.L. Riha*}

Depts of *Respiratory Medicine and "Radiology, Royal Infirmary Edinburgh, Edinburgh, UK.

Correspondence: R.L. Riha, Dept of Respiratory Medicine, Royal Infirmary Edinburgh, 51 Little France Crescent, Edinburgh, EH164SA, UK. E-mail: rlriha@hotmail.com

Statement of Interest: None declared.

\section{REFERENCES}

1 Lazarus AA, Thilagar B, McKay SA. Allergic bronchopulmonary aspergillosis. Dis Mon 2008; 54: 547-564.

2 Shah A. Aspergillus-associated hypersensitivity respiratory disorders. Indian J Chest Dis Allied Sci 2008; 50: 117-128.

3 Sher TH, Schwartz HJ. Allergic Aspergillus sinusitis with concurrent allergic bronchopulmonary Aspergillus: report of a case. J Allergy Clin Immunol 1988; 81: 844-846.

4 Schwartz HJ, Witt WJ, Sher TH. Allergic bronchopulmonary aspergillosis and allergic aspergillus sinusitis: case report. Ann Allergy 1992; 69: 447-448.

5 Braun JJ, Pauli G, Schultz P, et al. Allergic fungal sinusitis associated with allergic bronchopulmonary aspergillosis: an uncommon sinobronchial allergic mycosis. Am J Rhinol 2007; 21: $412-416$.

6 Erwin GE, Fitzgerald JE. Case report: allergic bronchopulmonary aspergillosis and allergic fungal sinusitis successfully treated with voriconazole. J Asthma 2007; 44: 891-895.

7 Shah A, Panchal N, Agarwal AK. Concomitant allergic bronchopulmonary aspergillosis and allergic Aspergillus sinusitis: a review of an uncommon association. Clin Exp Allergy 2001; 31: 1896-1905.

8 Shah A, Panjabi C. Contemporaneous occurrence of allergic bronchopulmonary aspergillosis, allergic Aspergillus sinusitis, and aspergilloma. Ann Allergy Asthma Immunol 2006; 96: 874-878.

9 Judson MA, Stevens DA. Current pharmacotherapy of allergic bronchopulmonary aspergillosis. Exp Opin Pharmacother 2001; 2: 1065-1071.

10 Wark PA, Gibson PG, Wilson AJ. Azoles for allergic bronchopulmonary aspergillosis associated with asthma. Cochrane Database Syst Rev 2004; 3: CD001108.

11 NHS. National Institute for Clinical Excellence. Asthma (uncontrolled) - omalizumab. 2007. Available from www.nice.org.uk/ TA133. 